\title{
Chapter 9 \\ Preliminary Observations \\ of Female-Female Bridging Behavior \\ in Tibetan Macaques (Macaca thibetana) \\ at Mt. Huangshan, China
}

\author{
Grant J. Clifton, Lori K. Sheeran, R. Steven Wagner, Jake A. Funkhouser, \\ and Jin-Hua Li
}

\subsection{Introduction}

Several species of the genus Macaca engage in an affiliative behavior commonly referred to as "bridging." Bridging is a triadic behavior in which two older individuals lift and hold an infant or juvenile between them while teeth chattering and/or licking the infant/juvenile's genitals (Ogawa 1995a). Bridging and similar affiliative

\section{G. J. Clifton (ه)}

Primate Behavior and Ecology Program, Central Washington University, Ellensburg, WA, USA

\section{K. Sheeran}

Primate Behavior and Ecology Program, Central Washington University, Ellensburg, WA, USA

Department of Anthropology and Museum Studies, Central Washington University, Ellensburg, WA, USA

e-mail: SheeranL@cwu.edu

R. S. Wagner

Department of Biological Sciences, Central Washington University, Ellensburg, WA, USA e-mail: WagnerS@cwu.edu

\section{J. A. Funkhouser}

Primate Behavior and Ecology Program, Central Washington University, Ellensburg, WA, USA

Department of Anthropology, Washington University in St. Louis, St. Louis, MO, USA

e-mail: jakefunkhouser@wustl.edu

J.-H. Li

School of Resources and Environmental Engineering, Anhui University, Hefei, Anhui, China International Collaborative Research Center for Huangshan Biodiversity and Tibetan Macaque Behavioral Ecology, Anhui, China

School of Life Sciences, Hefei Normal University, Hefei, Anhui, China e-mail: jhli@ahu.edu.cn 
triadic behaviors have been recorded in Barbary (M. sylvanus; Deag and Crook 1971; Taub 1984; Paul et al. 1996; Kubenova et al. 2017), stump-tailed (M. arctoides; Estrada and Sandoval 1977; Estrada and Estrada 1984), Assamese (M. assamensis; Kubenova et al. 2017), and Tibetan macaques (M. thibetana; Ogawa 1995a, b, c; Zhao 1996; Bauer et al. 2013). Adult males' bridging tends to occur in non-agonistic contexts and is often followed by other affiliative behaviors such as grooming (Ogawa 1995a, b; Deag and Crook 1971). Adult males of all species use younger male infants as the bridge more often than female infants and juveniles of both sexes (Ogawa 1995a; Deag 1980). In M. sylvanus, subordinate, lower-ranked males often bring an infant to a dominant individual to engage in affiliative behaviors (Deag and Crook 1971; Deag 1980). Deag and Crook (1971) hypothesized that subordinate males use infants as an "agonistic buffer" when initiating interactions with dominant males in order to reduce the probability of aggression. Alternatively, Taub (1984) proposed that these interactions were related to paternal caretaking and instead suggested the "enforced babysitting" hypothesis. Rather than using the infant as a buffer, Taub suggested that natal males in M. sylvanus use infants that are matrilineally related to them in order to develop bonds with infants and to inform others of their relatedness to the individual (1984).

Paul, Kuester, and Arnemann (1996) tested both the agonistic buffer and enforced babysitting hypotheses in dyadic male-infant interactions and triadic male-infant interactions (i.e., bridging) in $M$. sylvanus. DNA evidence conflicted with the enforced babysitting hypothesis by demonstrating that males did not interact with infants related to them and did not gain any additional reproductive benefits from interacting with particular females' infants. Conversely, frequency of male-infant interactions increased during intervals of high male-male tension, which supported the agonistic buffering hypothesis in M. sylvanus.

Ogawa (1995a, b, c) investigated male-male bridging behavior in Tibetan macaques (M. thibetana) and tested the agonistic buffering and enforced babysitting hypotheses. Ogawa found that bridging almost always occurred in non-agonistic contexts and that the frequency of bridging between males positively correlated with rates of grooming (Ogawa 1995a). Subordinate males initiated bridges with dominant males more often than vice versa, and initiating males were more likely to use the infants that were preferred in social interactions by dominant males. Aggression never occurred in male-male dyadic interactions in which a bridge took place but occasionally happened in non-bridging dyadic interactions. Ogawa (1995a) suggested that in macaque species with a higher than average socionomic sex ratio, such as Barbary and Tibetan macaques, males may use bridging to reduce social tensions caused by increased male-male competition and to reduce the possibility of aggression in future interactions. While Ogawa's (1995a) findings did not support the enforced babysitting hypothesis, natal group males did not prefer to bridge with infants related to them, suggesting that male-male bridging is not related to kinship. Adult males preferred to use male infants rather than female infants, and individual males had particular immature males that they used in bridging interactions more often than they used others. However, there was no evidence to suggest that infants gained any direct benefits from bridging: male and female infants experienced equal mortality rates despite the sex bias toward male infants in male-male bridging. 
Although all age and both sex classes engage in bridging, there has been little mention of bridging interactions involving females. Ogawa (1995c) studied bridging interactions between adult males and adult females. In contrast to the trend in malemale bridging, higher-ranked males were more likely to initiate male-female bridging interactions than were lower-ranked males. Additionally, males bridged more females they were in consort with. Ogawa (1995c) concluded that male-female bridging likely facilitates mating bonds.

Female-female bridging has been reported in M. thibetana (Ogawa 2006; Bauer et al. 2013) and M. arctoides (Estrada and Estrada 1984), and similar triadic femalefemale-infant interactions have been described in M. sylvanus (Deag and Crook 1971), but no studies have investigated female-female bridging in detail. When considering the existence of maternal relatedness and strong matrilines among females as well as the differing forms of within-sex competition between males and females, it is possible that female-female bridging does not serve the same purpose as male-male bridging. Moreover, maternity and females' matrilineal relationships may influence how they bridge with one another.

This study describes and analyzes female-female bridging behavior in a group of habituated Tibetan macaques and compares female-female bridging behavior to previous studies on male-male bridging behavior. We predicted female-female bridging would differ from male-male bridging and that these differences might aid in development of further hypotheses to explore female-female bridging in this species.

\subsection{Methods}

We conducted observations from 01 August to 21 September 2014 at the Valley of Wild Monkeys tourist site near Mt. Huangshan, Anhui Province, China $\left(30^{\circ} 29^{\prime} \mathrm{N}\right.$, $118^{\circ} 11^{\prime} \mathrm{W}$ ). One group of Tibetan macaques known as Yulingkeng A1 (YA1) ranges in the forest area surrounding platforms built to facilitate tourism. YA1 monkeys are free-ranging but are habituated to human presence and are provisioned with corn several times a day by park guards. Researchers have tracked and recorded matrilineal kinship, births, immigrations, and deaths since 1985 (Wada and Xiong 1996). At the study's start, the group consisted of 41 individuals: 9 adult males, 8 adult females, 4 subadult males, 4 subadult females, 6 juvenile males, 7 juvenile females, 2 male infants, and 1 female infant; however, the female infant died on 15 August 2014 (Table 9.1). We classified females as adult if they had given birth to least one offspring and were $\geq 6$ years old and as subadults if nulliparous and 4-5 years old. We classified infants as under 1 year of age, with the two male infants being roughly 2 months of age and the sole female infant being roughly 5 months of age at the start of the study. To establish a dominance hierarchy for use in this investigation, Lori K. Sheeran (LKS) collected dominance interaction behavioral data from 14 July to 27 August 2014 (36 days) from 7:00 to 12:00 and 14:00 to 17:00 daily. LKS utilized all-occurrence sampling to collect dominance data (Altmann 1974). Agonistic data 


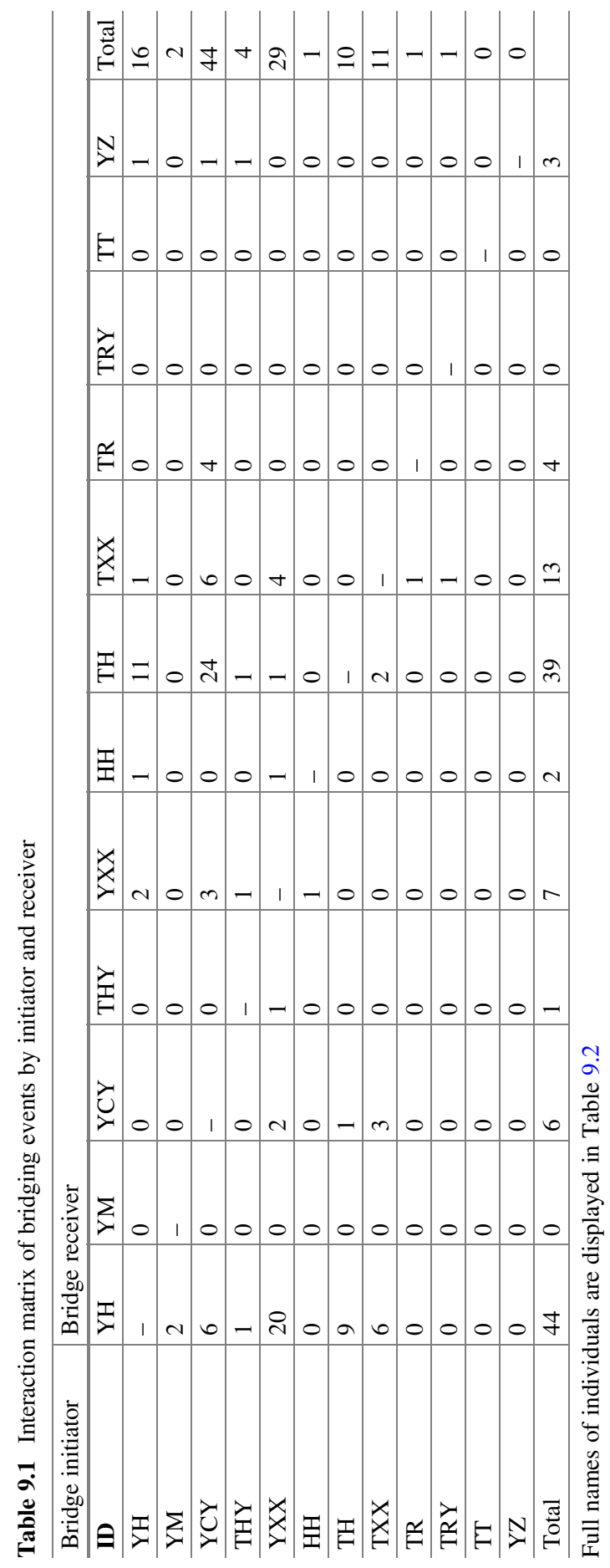


Table 9.2 Select results and demographics per individual

\begin{tabular}{|c|c|c|c|c|c|c|}
\hline $\begin{array}{l}\text { Female } \\
\text { individual }\end{array}$ & $\begin{array}{l}\text { Date of } \\
\text { birth }\end{array}$ & $\begin{array}{l}\text { Age } \\
\text { group }\end{array}$ & $\begin{array}{l}\text { Elo } \\
\text { score }^{\mathrm{a}}\end{array}$ & $\begin{array}{l}\text { Focal time } \\
\text { (hour) }\end{array}$ & $\begin{array}{l}\text { Bridge } \\
\text { initiations/h }\end{array}$ & $\begin{array}{l}\text { Bridge } \\
\text { receptions/h }\end{array}$ \\
\hline $\begin{array}{l}\text { YeXiaXue } \\
(\mathrm{YXX})\end{array}$ & $2010-05$ & Subadult & 1316 & 2.83 & 2.826 & 0.354 \\
\hline $\begin{array}{l}\text { HuaHong } \\
(\mathrm{HH})\end{array}$ & 2003-?? & Adult & 1173 & 2.00 & 0.498 & 0 \\
\hline $\begin{array}{l}\text { YeChunYu } \\
\text { (YCY) }\end{array}$ & 2009-03 & Subadult & 1172 & 3.13 & 2.874 & 0 \\
\hline YeHong (YH) & 2003-?? & Adult & 1136 & 3.53 & 1.134 & 1.416 \\
\hline YeMai (YM) & 1990-04 & Adult & 1096 & 2.23 & 0.45 & 0 \\
\hline TouRui (TR) & 2004-?? & Adult & 1057 & 2.53 & 0 & 0.396 \\
\hline $\begin{array}{l}\text { TouHong } \\
\text { (TH) }\end{array}$ & 2003-?? & Adult & 987 & 2.72 & 1.464 & 2.196 \\
\hline $\begin{array}{l}\text { TouXiaXue } \\
\text { (TXX) }\end{array}$ & $2008-03$ & Adult & 885 & 3.13 & 0.318 & 0.96 \\
\hline $\begin{array}{l}\text { TouRongYu } \\
\text { (TRY) }\end{array}$ & $2009-03$ & Subadult & 838 & 1.00 & 0 & 0 \\
\hline TouTai (TT) & 1991-04 & Adult & 766 & 2.23 & 0 & 0 \\
\hline YeZhen (YZ) & $1992-01$ & Adult & 729 & 2.97 & 0 & 0.336 \\
\hline $\begin{array}{l}\text { TouHuaYu } \\
\text { (THY) }\end{array}$ & 2009-04 & Subadult & 656 & 2.67 & 0.378 & 0 \\
\hline Mean & & - & - & 2.58 & 0.828 & 0.474 \\
\hline Median & & - & - & 2.70 & 0.414 & 0.168 \\
\hline
\end{tabular}

${ }^{a}$ We used Elo-rating scores as a metric of dominance status. Larger Elo scores indicate higher dominance status

consisted of fear-grin, scream, flee, displace, threat, lunge, chase, grab, slap, and bite as defined by Berman et al. (2004). Winners of these interactions were defined by either the actor in directional agonism or the recipient of a submissive behavior. Losers of these interactions were defined by those receiving directed agonism or the actors of submission. To derive a ranking order and dominance score for each individual, we analyzed these data using Elo-rating procedures (Neumann et al. 2011) in R ( $\mathrm{R}$ Core Team 2016).

We established interobserver agreement of $100 \%$ for all adult male and female, subadult female, and infant identities on 08 August 2014. We identified juveniles by the presence of their mother or their age and sex rather than individual identities due to the difficulty of learning individual juvenile identities within the limited timeframe of our study. We collected data from 8:00 to 12:00 and 13:00 to 18:00. To record bridging events, Grant J. Clifton (GJC) used 2-min focal samples (Altmann 1974) of adult and subadult females. We selected focal order randomly each day using a random number generator. Within focal samples, we recorded behaviors from an ethogram (Ogawa 1995a). Proximity refers touching or within arm's length (Sheeran et al. 2010). GJC also used all-occurrence sampling (Altmann 1974) to record bridges occurring outside of focal samples. If a bridge occurred or was suspected to occur between non-focal individuals, then the current 2-min focal 

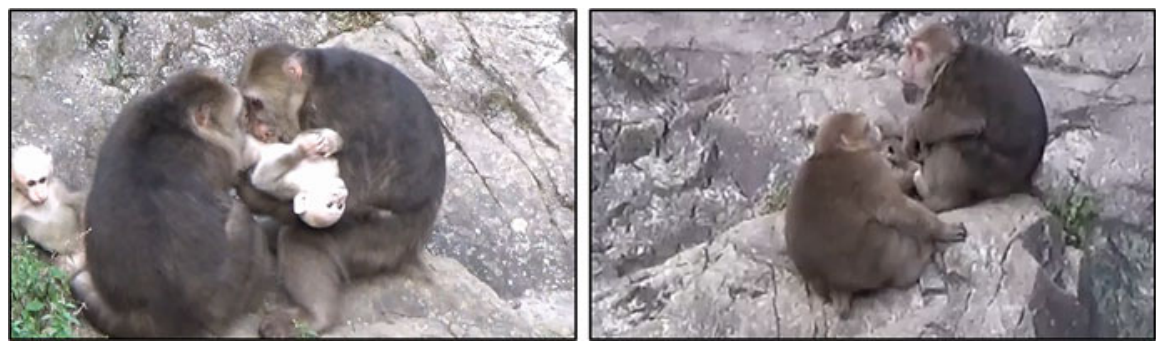

Fig. 9.1 Successful (left) and failed (right) bridge initiations (photo credit: Anne Salow)

was suspended, and the interacting dyad was observed for 2 min or until one individual left proximity. If a bridge occurred during a focal, we extended the focal for two additional minutes after the bridge to record context, but did not include the additional focal time in the focal data.

We used an ethogram modified from Ogawa (1995a) to record affiliative behaviors that often occurred before and/or during bridging. A bridge was defined as two individuals holding an infant or juvenile between one another with at least one individual teeth chattering or genital licking the infant. The bridge initiator was considered the individual who first teeth chattered or genital licked while lifting the infant or juvenile. A bridge began when both the initiator and receiver held up an infant. The bridge ended either when one of the individuals put down the infant or when no affiliative behaviors continued (other than grooming). Bridges that occurred within short succession of one another were considered separate events as long as at least one individual stopped holding the infant in between events.

We considered a bridge successful when the receiver held the infant or juvenile in the bridging position after receiving an initiation (Fig. 9.1). If a receiver teeth chattered or genital licked but turned away while the initiator was attempting a bridge, then it was considered failed (Fig. 9.1). Following Ogawa (1995a), we classified as Type I bridges in which the initiator brings an infant to a receiver or as Type II if the initiator approaches a receiver who is holding an infant (Fig. 9.2). Ogawa also observed bridges in which both males simultaneously approached and grabbed a single infant. In his study, he classified them as Type III bridges; we, on the other hand, did not observe Type III occur among females, so they were left out of our analysis.

We analyzed bridging behavior by initiator, receiver, and dyad. For individual and group comparisons, we used bridges recorded from focal animal sampling and converted bridges to rates to account for the uneven distribution of focal times. To calculate the rate of bridge initiations and bridge receptions per individual, we divided the number of bridge initiations and receptions from within the individual's focal samples by the total focal time for that individual. To calculate the rate of bridging in dyadic interactions, we calculated the total number of initiations by each individual and divided by the sum of the total focal follows for both individuals. Since focal and all-occurrence bridging dyads were significantly correlated (Kendall's tau; $\tau=0.65, n=22, p<0.01$ ) and since the proportions of Type I 

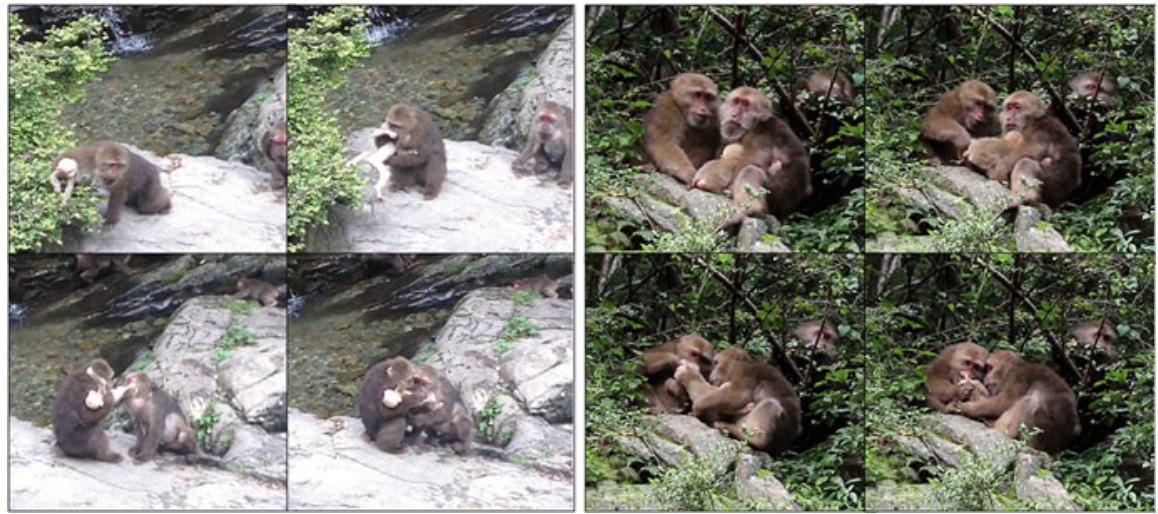

Fig. 9.2 Type I (left) and Type II (right) bridge initiations

and Type II bridges were not significantly different between focal and all-occurrence sampling (Fisher's exact test; $p>0.05, d f=2$ ), we pooled both sample sets for categorical analyses of bridge initiation type. However, we only included bridges recorded via focal animal sampling in our analysis of success rates. Because of our limited size and aim (to describe and categorize female-female bridging), we mainly used descriptive and simple parametric statistics. We used Pearson's correlation coefficient to analyze the relationship between dominance status (Elo scores) and bridge initiation/reception rates, paired-sample $t$ tests to examine differences in initiations rate by subordinate or dominant individual within each dyad, chi-square tests of independence, Fisher's exact, and binomial tests to investigate differences in the distribution of nonparametric frequencies of bridging event types.

\subsection{Results}

We recorded a total of $31 \mathrm{~h}$ of focal data and 119 bridging events (27 Type I; 92 Type II; 76 successful and 43 unsuccessful; Table 9.1). All four subadults and seven of eight adults were observed involved in at least one bridge (Table 9.1). Overall, we recorded 119 bridging events, $46(38.7 \%)$ of which were recorded via focal sampling.

Of all observed bridging events, we recorded 27 (22.7\%) that occurred via Type I initiation and 90 occurred via Type II initiation, of which $76(63.9 \%)$ were successful bridges and $43(36.1 \%)$ were failed bridges. In focal animal sampling, we recorded $7(13.2 \%)$ via Type I initiations and $39(84.8 \%)$ via Type II initiations, of which $21(45.7 \%)$ were successful bridges and $25(54.3 \%)$ were failed bridges. Table 9.2 displays all bridging initiations and receptions for each possible dyad and focal times and bridging rates for adult and subadult females. 


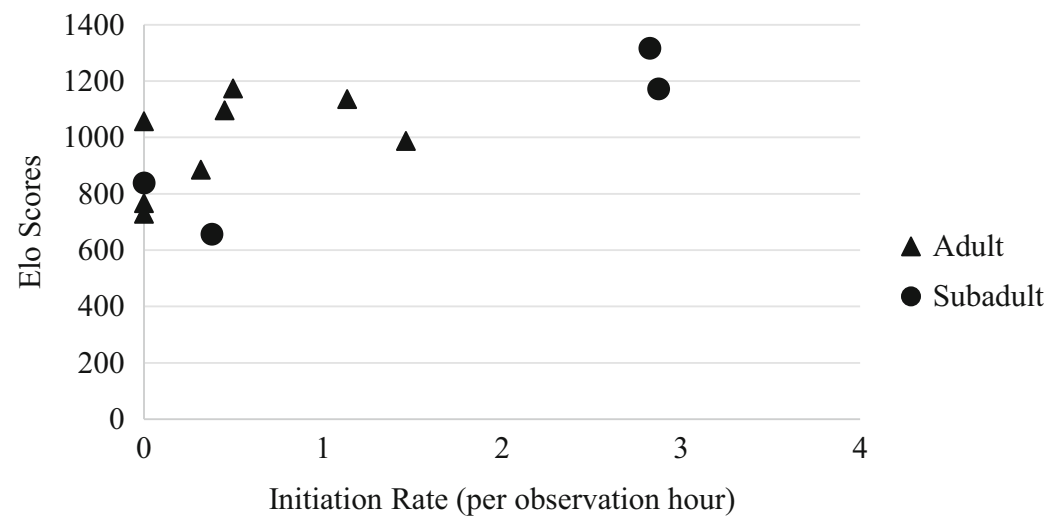

Fig. 9.3 Scatterplot of dominance status (Elo score) vs. bridge initiations (per hour of observation)

Female-female bridges were often subtle and did not involve audible vocalizations. Like male-male bridges, at least one female would lick the infant's genitals during bridges and sometimes touched the infant's genitals. Females often approached mothers who were holding infants and would peer at the infant before initiating the bridge by grabbing it. Successful bridges were never observed to be followed by aggression during our study, but receivers were occasionally observed committing aggression toward failed initiators.

The median bridge initiation rate for adults was 0.378 initiations per hour, whereas the median bridge initiation rate for subadults was 1.596 initiations per hour, but the sample size of the subadult group was too small to accurately test for significance. However, two subadult females, YCY and YXX, initiated far more often than any other adult or subadult (Table 9.1). The median bridge reception rate for adults was 0.366 receptions per hour, whereas no subadults were observed to be recipients of any bridging events. Like initiations, there were large differences in the number of bridges received by each individual within groups, with the two mothers of infants, $\mathrm{YH}$ and $\mathrm{TH}$, receiving a large majority of bridges (Table 9.1). Individuals who initiated bridges were not more likely to have their bridges reciprocated by those with whom they initiated $\left(r_{\mathrm{s}}=-0.45, n=17, p>0.05\right)$.

We analyzed the relationship between dominance status and bridge initiations using a Pearson's rank correlation. Social rank was positively correlated with bridge initiations $(r(12)=0.686, p=0.014$; Fig. 9.3), but social rank and bridge receptions were not significantly correlated $(r(12)=0.06, p=0.85$; Fig. 9.4).

To test for significance differences between the mean rates of bridge initiations by either dominant or subordinate individuals within each dyad, we used a pairedsamples $t$ test. In contrast to what has been reported for males, within each dyad, subordinate individuals did not initiate bridges significantly more than that dominant individuals. We found no significant difference between the mean rate of bridge initiation between dominant or subordinate individuals $(t(65)=1.398, p=0.167$, Fig. 9.5). While this difference is not significant, descriptively, we found that 


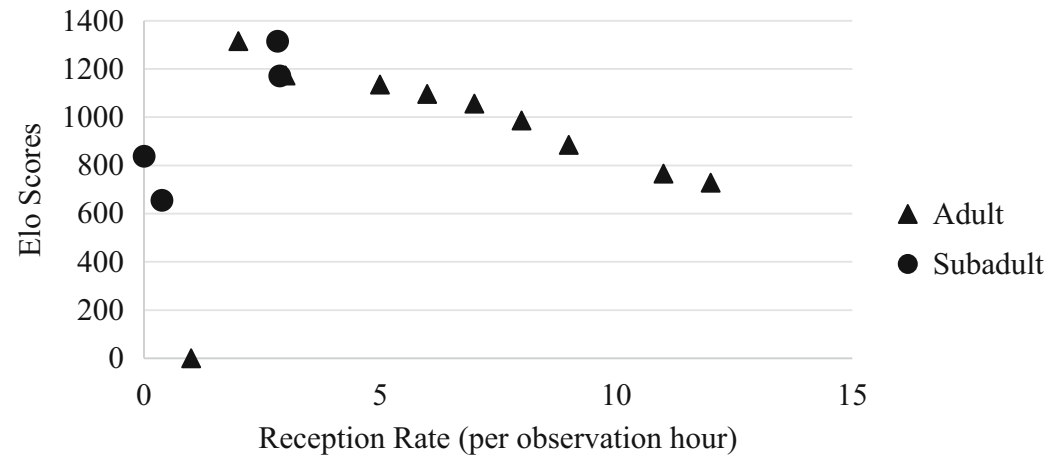

Fig. 9.4 Scatterplot of dominance status (Elo score) vs. bridge receptions (per hour of observation)

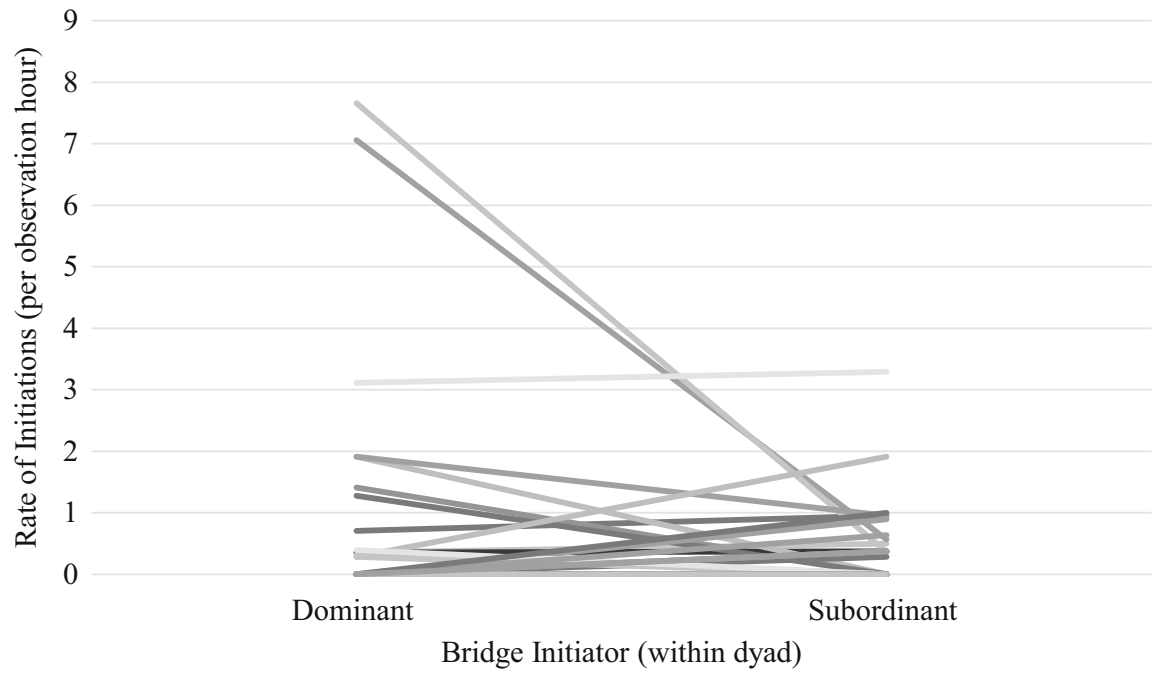

Fig. 9.5 Bridge initiation rates for the dominant vs. subordinate individual within each dyad. Each line represents a single dyad for a total of 22 dyads

dominant individuals were found to initiate bridges more $(0.415 \pm 1.35$ initiations per observation hour) than subordinate individuals $(0.195 \pm 0.52$ initiations per observation hour).

A majority of bridges occurred via Type II interactions. In bridges observed via focal sampling, all Type I bridges occurred by adults. Subadult females were observed initiating Type I interactions via all-occurrence sampling, but Type I interactions were still more likely to be initiated by an adult than a subadult (Fisher's exact test; $p<0.05, d f=1$; Fig. 9.6). Of all but two Type II bridges, the receiver of the bridge was the mother of the infant or juvenile being used, and in the two exceptions, the same subadult female, YXX, was the receiver. Similarly, in all but two Type I interactions initiated by adults, the initiator was holding her own 


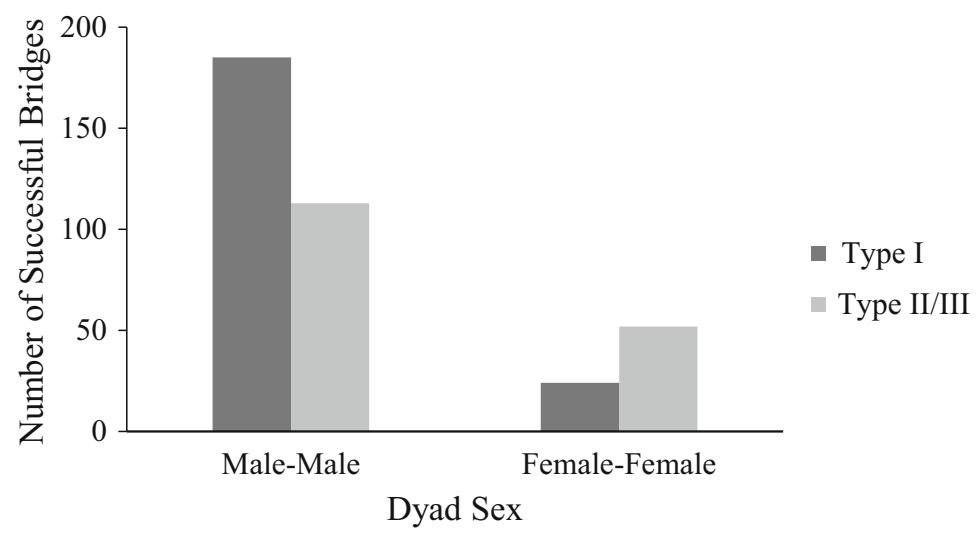

Fig. 9.6 Bar graph of successful Type I and Type II/III initiations between male and male

offspring. These two exceptions were initiated by the same individual, TXX, using TH's infant in both cases.

In Ogawa's (1995a) study of male-male bridging, 185 (62.1\%) bridges occurred via Type I initiations, 70 (23.5\%) occurred with Type II initiations, and 43 (14.4\%) occurred via Type III interactions. We did not observe female-female bridging via Type III initiations, so to compare males and females, we pooled Type II and Type III male bridges together for a total of 185 (62.1\%) Type I and 113 (37.9\%) Type II/III interactions. Since Ogawa (1995a) did not mention the occurrence of failed male-male bridge initiations, we only used successful female-female bridge initiations to compare differences between male-male and female-female bridges to ensure validity between our dataset and Ogawa's. Of successful female-female bridges, 24 (31.6\%) occurred via Type I initiations, and 52 (68.4\%) occurred via Type II initiations. Even after pooling the male Type II and Type III initiations together, which creates a more conservative estimate than if Type III initiations were excluded, females were still significantly more likely than males to use Type II bridge initiations over Type I initiations (chi square test for independence; $\chi^{2}=28.85, d f=1, p<0.01$; Fig. 9.6).

Bridges initiated by adults were significantly more likely to be successful than bridges initiated by subadults $(\varphi=0.46, d f=1, p<0.01)$. In focal samples, all Type I bridges were successful, but we recorded two failed Type I bridge initiations via all-occurrence sampling. However, these two failed initiations were from a subadult. Receivers showed no significant preference in accepting bridges that utilized either infants or juveniles, and we found no significant difference in success rates of bridges that were preceded by grooming vs. those that were not preceded by grooming, although successful bridges were significantly more likely than unsuccessful bridges to be followed by grooming $(\varphi=0.45, d f=1, p<0.01)$.

The two surviving infants in the group were used as a bridge in 88 out of 103 bridging cases, which is significantly more often than expected based on the proportions of infants and juveniles in the group (exact binomial test, $d f=1$, 


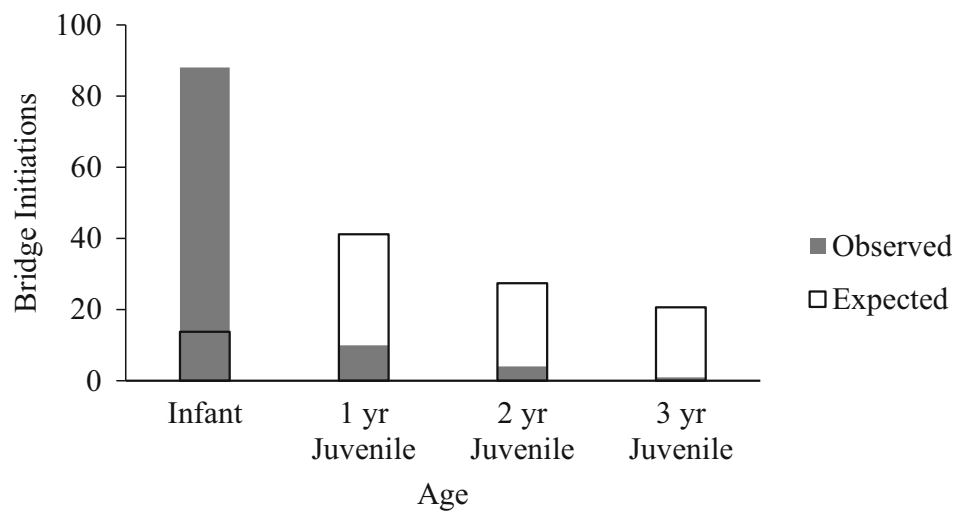

Fig. 9.7 Observed vs. expected use of infants and juveniles in bridge initiations after the death of TR's infant ( $n=103)$; expected values are calculated based on the proportion of individuals in each age group

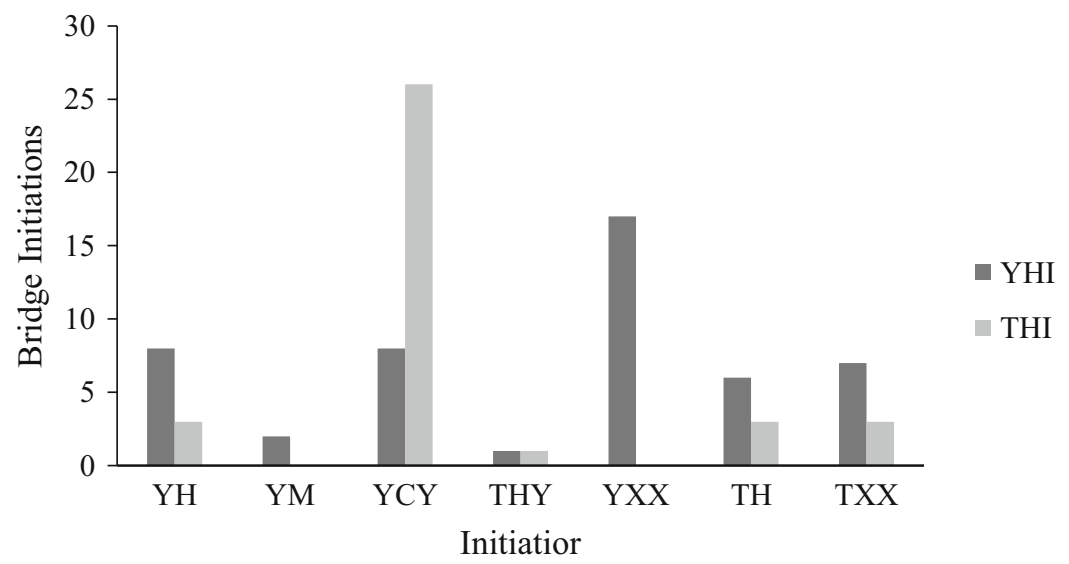

Fig. 9.8 Bar graph showing the number of bridge initiations using TH's infant (THI) and YH's infant (YHI) for each individual observed bridging with an infant at least once

$p<0.01$, Fig. 9.7). Of individuals who used infants to bridge, some individuals demonstrated a clear preference toward a specific infant over the other (Fisher's exact test; $d f=6, p<0.01$, Fig. 9.8), but our small sample size did not allow us to test the significance of the pairwise comparisons. 


\subsection{Discussion}

The agonistic buffering hypothesis was not supported among female-female bridges within our dataset for this group of Tibetan macaques. In contrast to what has been reported in males (Ogawa 1995a; Zhao 1996; Bauer et al. 2013), social rank was positively correlated with bridge initiations. Initiators were also not more likely to be subordinate to those who received their bridge attempts, which is evidence in contrast to the idea that subordinate females initiate bridges with dominant individuals to reduce the probability of future aggression (Ogawa 1995a). Further departing from the trends seen in males, females were not more likely to initiate bridges with those from which they received bridges. These data suggest the females in this group do not bridge in order to avoid conflicts, as has been observed for males.

Although there were no significant differences in bridging initiations between adult and subadult groups in this population, two of the subadult individuals, YCY and YXX, initiated far more bridges than any other individual. In contrast, the other two subadults, THY and TRY, rarely bridged. Interestingly, YCY and YXX were both much larger and more physically mature than either THY or TRY, who were both more juvenile-like in appearance and behavior. The mother that bridged the most out of the mothers without infant offspring, TXX, was relatively young for a mother and would normally be considered a subadult if not for the fact that she previously gave birth. This individual was also the only mother to initiate Type I bridges utilizing another mother's offspring and to initiate a Type II bridge toward a receiver holding her offspring. These bridges occurred toward subadult YCY.

As a group, adults received most of the recorded bridges, though statistically there was no difference between the rate at which adults and subadults were recipients. In particular, the infant mothers $\mathrm{TH}$ and $\mathrm{YH}$ each received far more bridges than any other individual. Out of bridges that involved adults, all except for two involved the mother of the infant. In addition, female bridges were more likely to occur by Type II initiation than by Type I. Ogawa (1995a) reported far more Type I initiations between males than Type II initiations. The comparison of female-female bridges and male-male bridges from Ogawa (1995a) demonstrates that this difference in Type I vs. Type II initiations between intra-sexed bridging events is significant, meaning that females are more likely to approach and initiate a bridge with a receiver who is holding an infant, whereas males are more likely to initiate while holding the infant. Of female-female Type II bridges, a large majority involved the mother as the receiver, and all of those that involved the mother as the receiver used her infant. Type I bridges were also more likely to be successful than Type II bridges, which may suggest an importance of the infant in the bridge rather than the relationship between the other individuals. In addition, individuals were not more likely to reciprocate bridges, which further suggests that the relationship between the older members of the bridging dyad is not as important as the infant. These trends support motherhood and interest in infants as factors in how female-female bridging is both initiated and received. 
Female-female bridging in our dataset did show some consistencies with malemale bridging, however. Similar to males, successful bridges never occurred in aggressive contexts and were not followed by aggression. Females also showed a bias toward initiating bridges with their own infants over other juveniles. Although initiators preferred to use infants, receivers did not appear to show a preference, as bridges initiated with juveniles were equally likely to be successful, though we should caution that the few observed juvenile bridges limits the power of our analysis. With that being said, it is nonetheless consistent with bridging trends between males observed in this same study group (Bauer et al. 2013). Moreover, among individuals that often bridged with infants, most showed a preference for one infant over the other. Interestingly, mothers of multiple offspring only initiated and received bridges utilizing their youngest offspring, further supporting the hypothesis that initiators are biased toward using younger individuals.

Unfortunately, the death of TR's infant, the only female infant, prevented us from investigating whether there are sex biases in the infants used in female-female bridges. YCY initiated four bridges using TR's infant, demonstrating that femalefemale bridges do occur with female infants, but we did not observe enough occurrences during this time for any sex biases to be tested. Of juvenile 1-yearolds, only females were used; however, there were five female 1-year-old juveniles and only one male 1-year-old juvenile. Moreover, TH was the mother of the single male 1-year-old juvenile, and she had a younger infant that she most often used in bridges. Without a more equally distributed sex ratio of infants and juveniles, it is impossible to state with these preliminary data whether or not females prefer to bridge with infants of a specific sex.

The preliminary results we present here can be used to generate future hypotheses regarding the function of bridging in females. In our dataset, the few individuals in the group make cross-comparisons between age classes difficult and limit a majority of the statistical analysis to less powerful, nonparametric, and descriptive procedures. Our study spanned a short time period, which limited the amount of focal data for each individual. This makes it unclear as to whether certain individuals never bridge or just do so less frequently than others. However, our study was conducted on the same group under similar conditions as past studies on bridging in male Tibetan macaques (Ogawa 1995a, b, c; Bauer et al. 2013; see also Zhao 1996 studying this species at Mt. Emei), which enabled us to directly compare malemale and female-female bridging behavior in Tibetan macaques within this particular group.

Despite the limitations, this study is the first to investigate bridging behavior between females in any species of macaque. Our results suggest that female bridging behavior may not follow the same pattern as male bridging and indicate that this behavior is related to female interest in infants than it is to the agonistic buffering hypothesis. Younger, higher-ranking female individuals may bridge more often because it allows them to gain alloparental experience from interacting with infants. Alternatively, female bridging may be a by-product of interest in mothers and infants, similar to what has been supported in female-infant interactions in bonnet macaques, M. radiata (Silk 1999). Future studies should follow the format of Ogawa 
(1995a) by observing bridges in relation to all female-female and female-infant interactions over the course of the entire year to enable a more definitive test of the agonistic buffering hypothesis.

Acknowledgments We thank Dao Zhang and the Chen family for logistical support while we were in China. We thank the members of the Huangshan Scenic Bureau for allowing us to conduct research at the site. We thank Anne Salow, Gregory Fratellone, and Sofi Bernstein for help with the data collection in the field and Dr. Dominic Klyve for assistance with statistical analysis. Grant J. Clifton particularly thanks Dr. Audrey Huerta and Dr. Linda Raubeson of CWU's Science Honors Research Program for their support. This research was funded by CWU's Science Honors Program and the National Science Foundation (OISE-1065589). Our research was reviewed and approved CWU's Institutional Animal Care and Use Committee (A011401).

\section{References}

Altmann J (1974) Observational study of behavior: sampling methods. Behaviour 49:277-266

Bauer B, Sheeran LK, Matheson MD, Li J, Wagner RS (2013) Male Tibetan macaques' (Macaca thibetana) choice of infant bridging partners. Zool Res 35(3):222-230

Berman CM, Ionica CS, Li JH (2004) Dominance style among Macaca thibetana on Mt. Huangshan, China. Int J Primatol 25(6):1283-1312

Deag JM (1980) Interactions between males and unweaned Barbary macaques: testing the agonistic buffering hypothesis. Behaviour 75:54-81

Deag JM, Crook JH (1971) Social behavior and "agonistic buffering" in the wild Barbary macaque Macaca sylvana L. Folia Primatol 15:183-200

Estrada A, Estrada R (1984) Female-infant interactions among free-ranging stumptail macaques (Macaca arctoides). Primates 25(1):48-61

Estrada A, Sandoval JM (1977) Social relations in a free-ranging troop of stumptail macaques (Macaca arctoides): male-care behavior I. Primates 18(4):793-813

Kubenova B, Konecna M, Majolo B, Smilauer P, Ostner J, Schulke O (2017) Triadic awareness predicts partner choice in male-infant-male interactions in Barbary macaques. Anim Cogn 20:221-232. https://doi.org/10.1007/s10071-016-1041-y

Neumann C, Duboscq J, Dubuc C, Ginting A, Irwan AM, Agil M, Widdig A, Engelhardt A (2011) Assessing dominance hierarchies: validation and advantages of progressive evaluation with Elo-rating. Anim Behav 82(4):911-921. https://doi.org/10.1016/j.anbehav.2011.07.016

Ogawa H (1995a) Bridging behavior and other affiliative interactions among male Tibetan macaques. Int J Primatol 16(5):707-729

Ogawa H (1995b) Recognition of social relationships in bridging behavior among Tibetan macaques (Macaca thibetana). Am J Primatol 35:305-310

Ogawa H (1995c) Triadic male-female-infant relationships and bridging behavior among Tibetan macaques (Macaca thibetana). Folia Primatol 64:153-157

Ogawa H (2006) Wiley monkeys: social intelligence of Tibetan macaques. Kyoto University Press, Kyoto

Paul A, Kuester J, Arnemann J (1996) The sociobiology of male-infant interactions in Barbary macaques, Macaca sylvanus. Anim Behav 51:155-170

R Core Team (2016) R: a language and environment for statistical computing. R Foundation for Statistical Computing, Vienna

Sheeran LK, Matheson MD, Li JH, Wagner RS (2010) A preliminary analysis of aging and potential social partners in Tibetan macaques (Macaca thibetana). In: Banak SD (ed) Collected readings in biological anthropology in honor of Professor LS Penrose and Dr. Sahrah B. Holt. Unas Letras Industria Editorial, Merida, pp 349-358 
Silk J (1999) Why are infants so attractive to others? The form and function of infant handling in bonnet macaques. Anim Behav 57:1021-1032

Taub DM (1984) Male caretaking behavior among wild Barbary macaques (Macaca sylvanus). In: Taub DM (ed) Primate paternalism. Van Nostrand Reinhold, New York, pp 20-55

Wada K, Xiong C (1996) Population changes of Tibetan monkeys with special regard to birth interval. In: Shotake T, Wada K (eds) Variations in the Asian macaques. Tokai University Press, Tokyo, pp 133-145

Zhao QK (1996) Male-infant-male interactions in Tibetan macaques. Primates 37(2):135-143

Open Access This chapter is licensed under the terms of the Creative Commons Attribution 4.0 International License (http://creativecommons.org/licenses/by/4.0/), which permits use, sharing, adaptation, distribution and reproduction in any medium or format, as long as you give appropriate credit to the original author(s) and the source, provide a link to the Creative Commons licence and indicate if changes were made.

The images or other third party material in this chapter are included in the chapter's Creative Commons licence, unless indicated otherwise in a credit line to the material. If material is not included in the chapter's Creative Commons licence and your intended use is not permitted by statutory regulation or exceeds the permitted use, you will need to obtain permission directly from the copyright holder. 